\title{
Bilateral superior vena cava with contralateral atrial drainage with brain abscess and transient ischemic attack in adult patient
}

\author{
Sheba John ${ }^{1}$, Santosh C Uppu ${ }^{1}$, Poyee P Tung ${ }^{2}$ and Dilachew Adebo ${ }^{*}$ \\ ${ }^{1}$ Department of Pediatrics, Division of Pediatric Cardiology, University of Texas Health Science Center at Houston, USA \\ ${ }^{2}$ Department of Internal Medicine, Division of Cardiovascular Medicine, University of Texas Health Science Center at Houston, USA
}

\begin{abstract}
Structured abstract

Objectives: Rare case of bilateral superior vena cava (SVC) with contralateral atrial drainage in an adult patient with brain abscess and transient ischemic attack diagnosed with cardiac magnetic resonance imaging (MRI).

Background: Anomalous systemic venous return is a variable congenital malformation. Furthermore, the consequence of anomalous systemic venous return is also variable. Prior literature has documented drainage of superior vena cava to the left atrium, with right to left shunting. We report a case of bilateral SVC with contralateral atrial drainage in an adult patient. The consequence of the right to left shunt resulted in brain and frequent episodes of transient ischemic attack (TIA).
\end{abstract}

Methods: Evaluation of echocardiography and cardiac MRI demonstrated bilateral SVC with contralateral atrial drainage.

Results: Transthoracic echocardiogram revealed dilated coronary sinus. Agitated saline bubble contrast study from right upper extremity showed immediate opacification of the left cardiac chambers suggestive of right SVC draining to left atrium. Transesophageal echocardiogram confirmed dilated coronary sinus; agitated saline contrast injection from left arm revealed complete opacification of the coronary sinus followed by opacification of the right heart, consistent with the presence of the left sided SVC draining to dilated coronary sinus. Cardiac MRI confirmed right SVC draining to the left atrium and the left SVC draining to the right atrium.

Conclusions: Anomalous systemic venous return is a relatively common and variable congenital malformation with heterogeneous consequences. Anomalous drainage of systemic venous return to the left atrium is an unusual variant of systemic venous return anomalies, which is characterized by right-to-left shunt. We present extremely rare case of bilateral SVC draining into contralateral atrium, resulting in a right-to-left shunt. This right-to-left shunt can result in persistent hypoxemia, mild cyanosis, paradoxical embolism with brain abscess. Patients may also remain asymptomatic. With accurate diagnosis, this condition can be treated with good short-term results.

\section{Condensed abstract}

Anomalous systemic venous return is a variable congenital malformation. Furthermore, the consequence of anomalous systemic venous return is also variable. Prior literature has documented drainage of superior vena cava (SVC) to the left atrium, with right to left shunting [1-3]. We report extremely rare case of bilateral superior vena cava with contralateral atrial drainage in an adult patient. The consequence of the right to left shunt in this patient resulted in brain abscess as well as frequent episodes of transient ischemic attack.

\section{Introduction}

Anomalous systemic venous return is a variable congenital malformation with a range of consequences. Prior literature has documented drainage of SVC to the left atrium, with right to left shunting [1-3]. We present a report extremely rare case of bilateral superior vena cava with contralateral atrial drainage in an adult patient. The consequence of the right to left shunt in this patient resulted in brain abscess as well as frequent episodes of transient ischemic attack. A 45-year-old male with no significant medical history presented with one-month history of intermittent blurry vision and headache, followed by severe headache with neck stiffness, nausea and vomiting. MRI brain demonstrated a ring enhancing mass with restricted diffusion consistent with a brain abscess. He subsequently underwent right occipital craniotomy with evacuation of intracerebral abscess. A peripherally inserted central line was placed from right upper extremity, intravenous antibiotics was started, and he was eventually discharged home. He presented a number of times over the next weeks for transient episodes of left sided hemiparesis and slurred speech, often occurring with the administration of his intravenous antibiotics. Repeat head imaging was unrevealing apart from postoperative surgical

${ }^{\star}$ Correspondence to: Dilachew Adebo, Department of Pediatrics, Division of Pediatric Cardiology, University of Texas Medical School at Houston, 6410 Fannin Street, Suite 425, Houston, Texas 77030, USA, Tel: 001773627 9497, E-mail: Dilachew.A.Adebo@uth.tmc.edu

Key words: bilateral SVC, anomalous systemic venous return, transient ischemic attack, magnetic resonance imaging, echocardiography

Received: September 07, 2020; Accepted: September 18, 2020; Published: September 28, 2020 

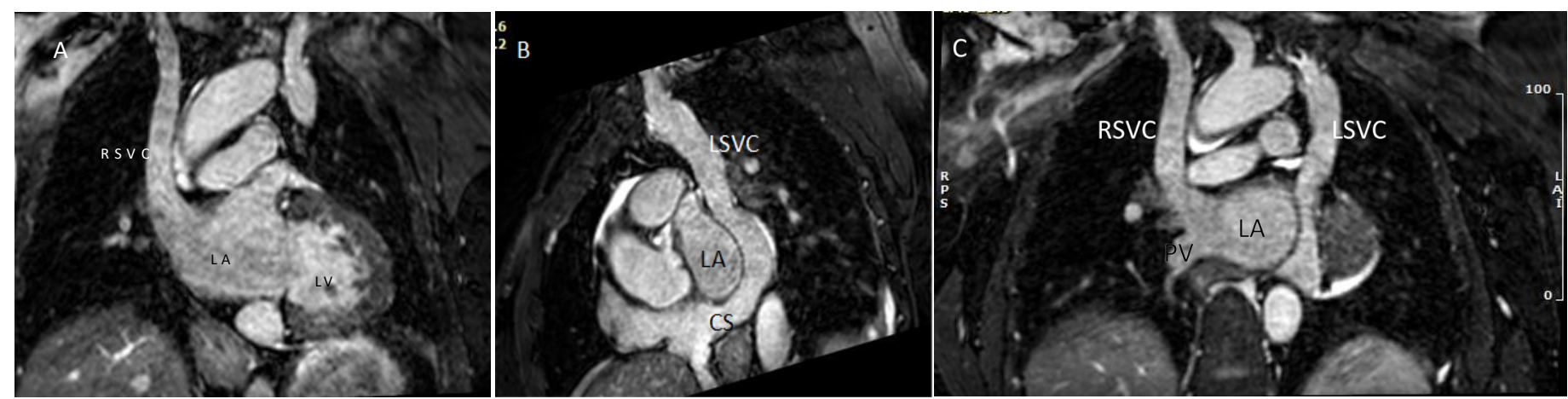

Figure 1A, B \&C. Whole heart T2 prep MRI Imaging of 45-year-old adult patient showing bilateral superior vena cavae with right superior vena cava draining to left atrium and left superior vena cava draining to right atrium via dilated coronary sinus.

RSVC: Right superior vena cava; LSVC: Left superior vena cava; LA: Left atrium; LV: Left ventricle; RA: Right atrium; CS: Dilated coronary sinus; PV: Pulmonary vein

changes. On clinical examination, patient was noted to have clubbing of his fingernails and mild hypoxia, oxygen saturations were $90-94 \%$ on room air (Figure 1A, B and C).

\section{Methods}

Evaluation with echocardiography and cardiac MRI demonstrated bilateral SVC with contralateral atrial drainage.

\section{Results}

Transthoracic echocardiogram revealed dilated coronary sinus. Agitation saline bubble contrast from right upper extremity showed immediate opacification of the left cardiac chambers suggestive of right superior vena cava draining to left atrium. Transesophageal echocardiogram was performed which confirmed dilated coronary sinus; next, agitated saline bubble contrast injection from the left arm revealed complete opacification of the coronary sinus followed by complete opacification of the right heart, consistent with the presence of the left sided SVC draining to dilated coronary sinus. Cardiac MRI further confirmed findings of a right SVC draining to the left atrium and the left SVC draining to the right atrium (Figure 1A, B and C).

\section{Discussion}

Anomalous systemic venous return is a relatively common and variable congenital malformation with heterogeneous consequences. Persistent left sided superior vena cava with bilateral superior vena cava is a systemic venous anomaly with reported prevalence of up to $0.5 \%$ in the general population and up to $10 \%$ in patients with congenital heart disease [4]. However anomalous draining of systemic venous return to the left atrium is a rare variant of systemic venous return anomalies, which is characterized by right-to-left shunt. We present extremely rare anomaly of the systemic venous return with bilateral superior vena cava draining into contralateral atrium, resulting in a right-to-left shunt. This right-to-left shunt can result in persistent hypoxemia, mild cyanosis, and paradoxical embolism with brain abscess. With accurate diagnosis, this condition can be treated with good short-term results. Long term studies are lacking.

\section{Conflict of interest}

We know of no conflicts of interest associated with this publication and there has been no financial support for this work that could have influenced its outcome.

\section{References}

1. Van Praagh S, Geva T, Lock JE, Nido PJ, Vance MS, et al. (2003) Biatrial or left atrial drainage of the right superior vena cava: anatomic, morphogenetic, and surgical considerations-report of three new cases and literature review. Pediatr Cardiol 24: 350-363. [Crossref]

2. Alghamdi MH, Elfaki W, Al-Habshan F, Aljarallah AS (2015) Bilateral superior vena cava with right superior vena cava draining into left atrium. J Saudi Heart Assoc 27: 123-126. [Crossref]

3. Sakai C, Tetsuhiro Y, Tomonori M, Otsuka A, Kato Y, et al. (2017) Imaging of Rightto-Left Shunt in an Adult Patient with Unroofed Coronary Sinus with Persistent Left Superior Vena Cava: Diagnostic Clue and Pitfall. Int Heart J 58: 1008-1011. [Crossref]

4. Oppido G, Napoleone CP, Turci S, Giardini A, Formigari R, et al. (2006) Right superior vena cava draining in the left atrium: anatomical, embryological, and surgical considerations. Ann Thorac Surg 81: 2313-2315.

Copyright: (C2020 John S. This is an open-access article distributed under the terms of the Creative Commons Attribution License, which permits unrestricted use, distribution, and reproduction in any medium, provided the original author and source are credited. 